\title{
Multi-channel Scheduling Algorithm for Multi-subsystem-based \\ VPON in Metro-Access Optical Network
}

\author{
Weidong Xia, Chaoqin Gan*, Shucong Ma, Luxi Xu, Weilun Xie \\ Key Laboratory of Specialty Fiber Optics and Optical Access Networks \\ Shanghai University, 200072 \\ Shanghai, China \\ *Email: cqgan@shu.edu.cn
}

\begin{abstract}
In the metro-access optical network, a multi-channel scheduling algorithm is proposed for multi-subsystem-based virtual passive optical network (VPON) that can implement the syncretism of multiple systems such as time division multiplexing-PON (TDM-PON), wavelength division multiplexing-PON (WDM-PON), orthogonal frequency division multiplexing-PON (OFDM-PON) or their hybrid PON technologies. Through a module named adaptive transceiver, this algorithm can be directly applied in the metro segment with the reuse of original deployment in the access segment. The algorithm can be employed to schedule wavelength and subcarrier resource in WDM/TDM subsystems and WDM/OFDM subsystems respectively. Centralized management is used in the algorithm to facilitate channel scheduling and algorithm implementation. An optimal scheduling length is derived in the algorithm to realize the maximization of channel-utilized ratio. Two cases are investigated to demonstrate the effectiveness of the proposed algorithm in the channel-utilized ratio.
\end{abstract}

Keywords: virtual passive optical network (VPON), multiple subsystems, metro-access optical network, mutli-channel scheduling.

\section{Introduction}

Network virtualization is an effective solution to improve the utilization of network resource [1-3]. In the backbone network, the network virtualization such as virtual private network (VPN) has been deeply studied and widely used [4]. Through VPN, the intra-network is available for remote users. In the access network, passive optical network (PON) as its main technology has been employed [5, 6]. Thus, the research on virtualization of access network mainly aims at the virtualization of PON, i.e., virtual PON (VPON) [7-14]. VPON can realize the virtualization of resource and can be dynamically constructed based on the real applications and requirements [8-10]. So far, the studies on VPON are conducted from different perspectives. For example, a VPON-based FlexPON architecture is proposed in [8-10] to achieve the 
full service access. A hybrid wavelength division multiplexing (WDM)/time division multiplexing (TDM) PON with dynamic VPON capability is proposed in [11, 12]. A VPON based on orthogonal frequency division multiplexing (OFDM) is proposed in $[13,14]$. It supports various types of custom access experiments. The above studies are all adapted to the development of PON and existing three-layer (i.e., core layer, convergence layer and access layer) architecture of metro optical network. On the other hand, flattening is the inevitable trend of telecommunication network. To the metro optical network, the existing three-layer architecture will be evolved into the two-layer (i.e., core-layer and metro-access layer) architecture in the future $[15,16]$. Now the architectures and performance of metro-access optical network have been widely studied. For example, ref. [17] proposed a RPR-EPON-WiMAX hybrid network, including an architecture and a joint MAC protocol, as a solution for metro-access network bridging. Capacity and delay analysis of integrated metro-access networks has been studied in [18]. An OFDM-based metro-access network is proposed in [19] to enable scalable and reconfigurable all-optical VPN. In addition, the evolution to metro-access optical network will be smooth. However, existing access network technologies mainly include TDM-PON, WDM-PON, OFDM-PON, etc. It means these subsystems will be syncretized in metro-access optical network in the future. At present, the related research on multi-subsystem-based VPON (MS-VPON) in metro-access optical network (MAON) is little. So, the research is quite necessary. Since the implementation of the MS-VPON in MAON is a sophisticated process, original deployment in access segment should be reused as far as possible. It can not only facilitate network upgrade, but also reduce deployment cost. At this time, the key to implementing the network communication rises up to the metro segment. While in the metro segment, multi-channel scheduling is a key problem. Therefore, this paper mainly focuses on the multi-channel scheduling algorithm. The algorithm can be employed to schedule wavelength and subcarrier resource in WDM/TDM subsystems and WDM/OFDM subsystems respectively. Centralized management is used in the algorithm to facilitate channel scheduling and algorithm implementation. An optimal scheduling length is derived in the algorithm to realize the maximization of channel-utilized ratio. Simulation results demonstrate that the proposed algorithm obtains higher channel-utilized ratio.

\section{The MS-VPON in MAON}

A MS-VPON scenario in MAON is shown in Fig. 1. The corresponding programmable VPON architecture is illustrated in Fig. 2. Considering the smoothness of network evolution, this scenario contains a central node $(\mathrm{CN})$, several flexible 
control nodes (FCNs), a traditional optical line terminal (OLT), and a number of optical network units (ONUs). The CN, FCNs and OLT are distributed in the fiber ring. Each FCN (or OLT) connected with ONUs is in tree topology. The centralized management of the whole network is realized in the $\mathrm{CN}$ that is the unique node between core layer and metro-access layer. OLT and ONUs are traditional nodes in PON. OLT is responsible for providing service to ONUs. ONUs are used to make an access to users. As shown in Fig. 2, the OLT is consisted of transceiver and TDM media access control (MAC) (e.g., EPON MAC). They are the same components as the already deployed systems. In other words, the already deployed OLT will not be changed in terms of the hardware. Therefore, the compatibility with already deployed systems can be realized. The FCN as the intermediate node of the network has the functions of remote node $(\mathrm{RN})$ and OLT. It includes a wavelength-routed module and an advanced OLT. As shown in Fig. 2, the wavelength-routed module can route the wavelength from the $\mathrm{CN}$ to the advanced OLT. In addition, it can also route the wavelength to ONU and achieve the function of RN. At this time, the upstream/downstream communications are controlled by the CN. Thus, a WDM-PON in the metro-access network is formed. The advanced OLT is made up of adaptive transceivers, buffer and MAC group. The adaptive transceivers have the capability to transmit and receive data streams in different subsystems such as TDM-PON, OFDM-PON or their hybrid PON technologies. The MAC group can achieve the access control of these subsystems. Therefore, the already deployed ONUs can be admitted to the network through the MAC group and then communicate with $\mathrm{CN}$ through the adaptive transceiver. According to the above, the syncretism of multiple subsystems can be realized. In a word, a VPON can be made up of multiple subsystems such as WDM-PON, TDM-PON, OFDM-PON or their hybrid PON technologies.

Based on the content mentioned above, the difference of deploying this architecture can be divided into two parts. The first part is to achieve compatibility with already deployed systems, OLT is retained in the architecture. It is consisted of transceiver and TDM MAC as shown in Fig. 2. They are the same components as the already deployed systems. Therefore, original deployments can all be reused in this situation. The second part is to achieve the syncretism of multiple subsystems, newly-designed FCN is used in the architecture. Due to the MAC group in the FCN can achieve the access control of different PON systems, the deployment in the access segment can be retained in this situation while the deployment in the metro segment should be replaced by the FCN.

In addition, through deploying this architecture, capital expenditure (CAPEX) 
and operating expense (OPEX) can be dropped significantly. This is because that CAPEX mainly aims at the cost on infrastructure investment (i.e., network deployment). Because the deployment in the access segment can be reused, the cost of network deployment will be dropped dramatically and it will move up to the cost-insensitive metro segment. OPEX mainly focuses on the operating expense. Because VPON can be dynamically constructed based on the real applications and requirements, the network resource can be allocated flexibly. This will significantly improve the utilization of network resource. In other words, operating expense can be decreased obviously.

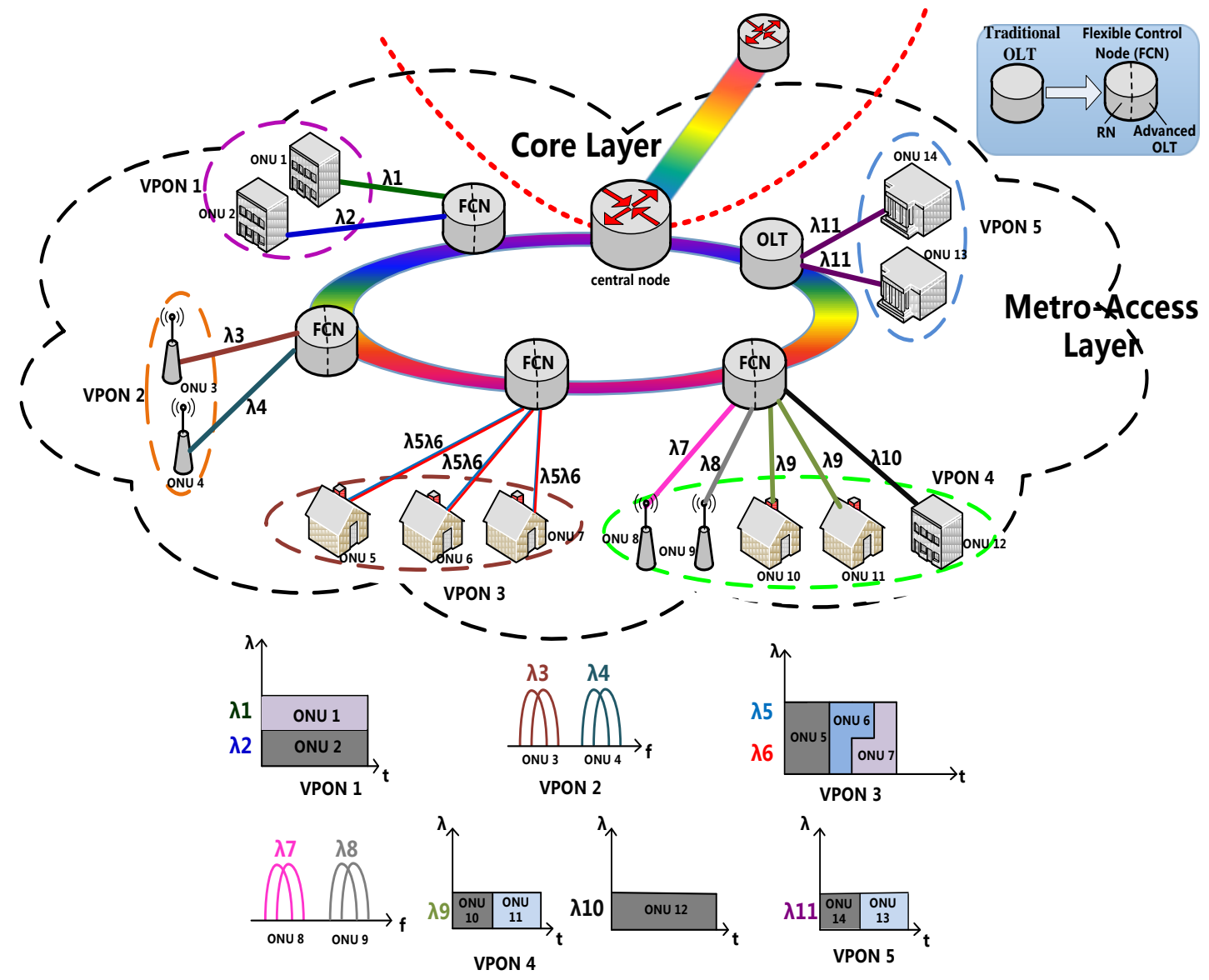

Fig. 1 The MS-VPON scenario 


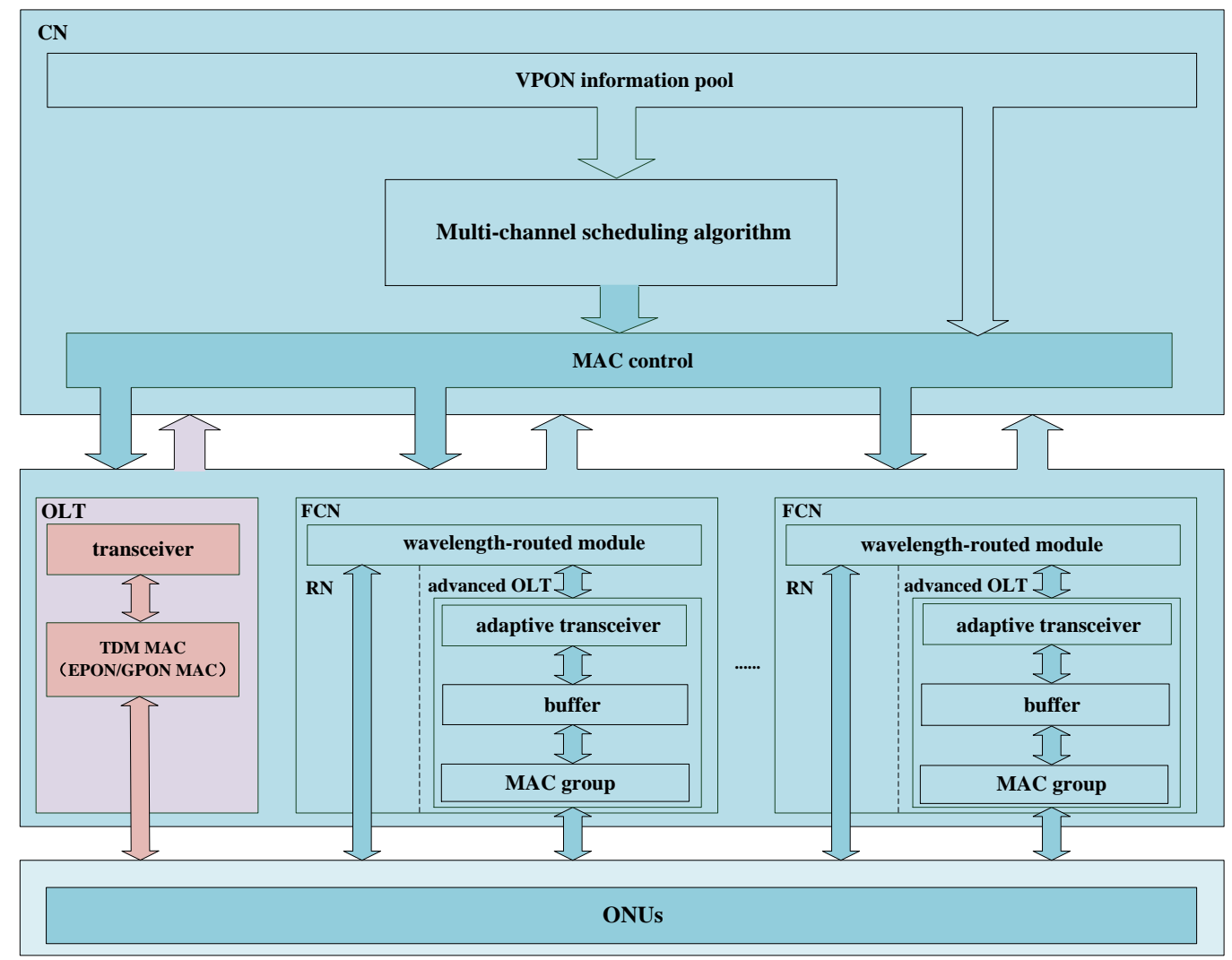

Fig. 2 The programmable VPON architecture

\section{Multi-channel scheduling algorithm}

As mentioned above, a VPON can be made up of multiple subsystems. Without loss of generality, we assume the VPON is made up of WDM/TDM subsystems or WDM/OFDM subsystems. To realize the multi-channel scheduling in the metro segment with the reuse of original deployment in the access segment, the adaptive transceiver is used. The function diagram of the adaptive transceiver is depicted in Fig. 3. The adaptive transceiver consists of two parts. The upper part is for WDM/TDM subsystems. The lower part is for WDM/OFDM subsystems. In each part, two pairs of transceivers are adopted due to the simultaneous transmission of downstream and upstream traffic. Among them, the transceiver in the right is used to realize the communication in the access segment, while the transceiver in the left is used to realize the communication in the metro segment.

The working principle of the adaptive transceiver is described as follows: in the downstream, the traffic from the $\mathrm{CN}$ will be firstly received by WDM/TDM-Rx or WDM/OFDM-Rx and then be distributed to the subsystems in the access segment by WDM/TDM-Tx or WDM/OFDM-Tx. In the upstream, the subsystem will send the corresponding traffic to the adaptive transceiver. Then, the traffic from the access segment will be received by WDM/TDM-Rx or WDM/OFDM-Rx and then be transported to the $\mathrm{CN}$ by WDM/TDM-Tx or WDM/OFDM-Tx. In this way, the tree 
wavelength of the access segment can be converted to the ring wavelength of the metro segment. Thus, original deployment in the access segment can still be used. Then, the key to implementing network communication rises up to the metro segment. Because the adaptive transceiver is included in the FCN, the communication between FCN and $\mathrm{CN}$ is a critical problem. In addition, due to the channel resource in the metro segment is limited [20], the multi-channel scheduling algorithm is employed to maximize the utilization of channel resource. Moreover, ring wavelength scheduling will be used in this paper. The reason is that as mentioned above, WDM/TDM subsystems will send the traffic to the adaptive transceiver. Then, the traffic from the access segment will be received by WDM/TDM-Rx and then be transported to CN by WDM/TDM-Tx. Because WDM/TDM-Tx is located in metro segment as shown in Fig. 3, its transmission wavelength will be ring wavelength. In addition, if tunable laser is used in WDM/TDM-Tx, a subsystem can be placed on multiple ring wavelengths. Therefore, to achieve the maximization of channel-utilized ratio, corresponding scheduling mechanism (e.g., ring wavelength scheduling) needs to be used in this paper.

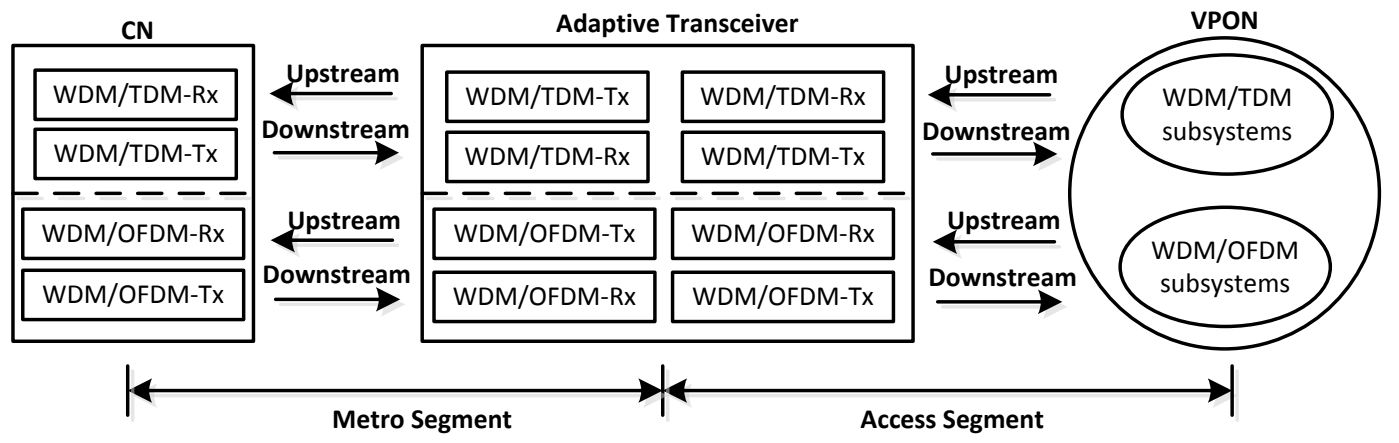

Fig. 3 The function diagram of the adaptive transceiver

The centralized management is used in the multi-channel scheduling algorithm to facilitate channel scheduling and algorithm implementation. Therefore, the multi-channel scheduling algorithm should be embedded in the $\mathrm{CN}$ as shown in Fig. 2 . The CN schedules wavelength and subcarrier resource and controls FCNs to execute the scheduling result. Fig. 4 shows the details of the above process as follows: 1) FCNs report the subsystems' demand to the CN. 2) Based on the available channel resource, the $\mathrm{CN}$ runs multi-channel scheduling algorithm to determine add/drop wavelengths and subcarriers at FCNs. 3) The CN sends the scheduling results to FCNs. Then, FCNs select right add/drop wavelengths and subcarriers when corresponding data arrives at FCNs. 4) Based on the scheduling results in 2), FCNs start the upstream transmission. 


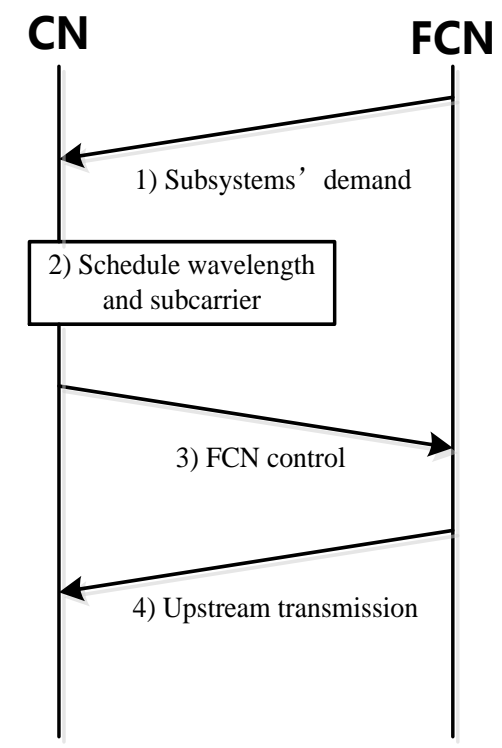

Fig. 4 The flowchart of multi-channel scheduling algorithm

In this paper, we focus on the design of a scheduling algorithm for maximizing channel-utilized ratio. To the WDM/TDM subsystems, the multi-channel scheduling algorithm is aimed at ring wavelengths scheduling. To the WDM/OFDM subsystems, the multi-channel scheduling algorithm is aimed at ring subcarriers scheduling. In other words, the channel refers to wavelength in WDM/TDM subsystems and refers to subcarrier in WDM/OFDM subsystems. The channel-utilized ratio is defined as follows:

$$
U=\frac{\sum_{i=1}^{N} d(i)}{L_{o p t} \cdot W}
$$

where $N$ is the total number of WDM/TDM subsystems or WDM/OFDM subsystems. $d(i)$ is the bandwidth demand of $i$ th WDM/TDM subsystem or WDM/OFDM subsystem. For simplicity, we assume $d(i)$ stands for the number of time slots requested by the subsystem. $L_{\text {opt }}$ is the optimal scheduling length. $W$ is the number of available channels.

\section{A. WDM/TDM subsystems}

To the WDM/TDM subsystems, we assume the transceiver in each FCN is tunable laser and tunable receiver, and its tunbale range can cover the $W$ channels. The wavelength tunability gives us the option to place a subsystem on multiple channels conditioned-on there being no time for overlapping among all allocated time slots from different channels for the same subsystem. We call this constraint the "non-overlapping constraint". 
As shown in Eq. (1), the smaller the scheduling length, the higher the channel-utilized ratio achieved. Therefore, the scheduling length is an important factor in the scheduling algorithm, and its establishment needs two steps. Firstly, to guarantee the allocated time slots can meet the demand of all subsystems, i.e., $L \cdot W \geq \sum_{i=1}^{N} d(i)$, a temporary scheduling length $L_{1}$ is defined as follows:

$$
L_{1}=\left\lceil\frac{\sum_{i=1}^{N} d(i)}{W}\right\rceil
$$

where $\lceil x\rceil$ is the minimum integer not smaller than $x$. Secondly, to satisfy the "non-overlapping constraint", the optimal scheduling length is defined as follows:

$$
L_{\text {opt }}=\max \left(\max (d(i)), L_{1}\right), 1 \leq i \leq N
$$

To maximize the channel-utilized ratio of WDM/TDM subsystems, the multi-channel scheduling algorithm is proposed here. The algorithm works as follows: 1) sort subsystem's demand $d(i)$ with descending order; 2) calculate the optimal scheduling length $L_{\text {opt }} ; 3$ ) assign subsystems to channels according to the decreasing order of their demand. The maximum number of time slots to be assigned in each channel is equal to the optimal scheduling length $L_{o p t}$. In the algorithm, the channel-utilized ratio is calculated as follows:

$$
U=\frac{\sum_{i=1}^{N} d(i)}{\max \left(\max (d(i)),\left[\frac{\left.\sum_{i=1}^{N} d(i)\right]}{W}\right]\right] \cdot W}
$$

According to the above process, we can know that the step 3) is the key point of the whole algorithm. To illustrate it, the pseudo-code of the step 3) is shown in Fig. 5. $a(j)$ is used to record how many time slots are allocated from the $j$ th channel and $S(i)$ is used to record the scheduling result for subsystem $i . S(i)$ is a set and it contains three elements, i.e., $C_{I D}, T_{s}, T_{e}$, where $C_{I D}$ is the channel identity, $T_{s}$ is 
the starting time slot and $T_{e}$ is the ending time slot. $k$ is the descending order of $d(i)$ among all $N$ subsystems' demand. $k=1$ means the subsystem has the maximum demand while $k=N$ means the subsystem has the minimum demand. $q(k)$ denotes the mapping from $k$ to $i$ and therefore its value equals $i$, i.e., $q(k)=i$.

The algorithm is described as follows: at first, the scheduling algorithm will set initial value for $a(j)$ and $S(i)$ (i.e., line 1 and line 2). Then, the algorithm starts with the first channel (i.e., line 3) to schedule the subsystems according to the descending order of their demand (i.e., line 4). After that, the mapping from $k$ to $i$ is done to start scheduling for subsystem $i$ whose descending order is $k$ (i.e., line 5). The scheduling algorithm needs each channel to allocate $L_{o p t}$ time slots at most. When it is time to schedule subsystem $i$ onto channel $j$, the algorithm needs to check whether channel $j$ has enough time slots for subsystem $i$ (i.e., line 6). If it does, the algorithm places subsystem $i$ onto this channel $j$ (i.e., line 7) and updates $a(j)$ (i.e., line 8); otherwise (i.e., line 9), the algorithm places subsystem $i$ onto two channels, $j+1$ and $j$ (i.e., line 10), and then updates $a(j+1)$ (i.e., line 11). At time, the channel $j$ arrives at the optimal scheduling length (i.e., line 12) and next scheduling will start with channel $j+1$ (i.e., line 13).

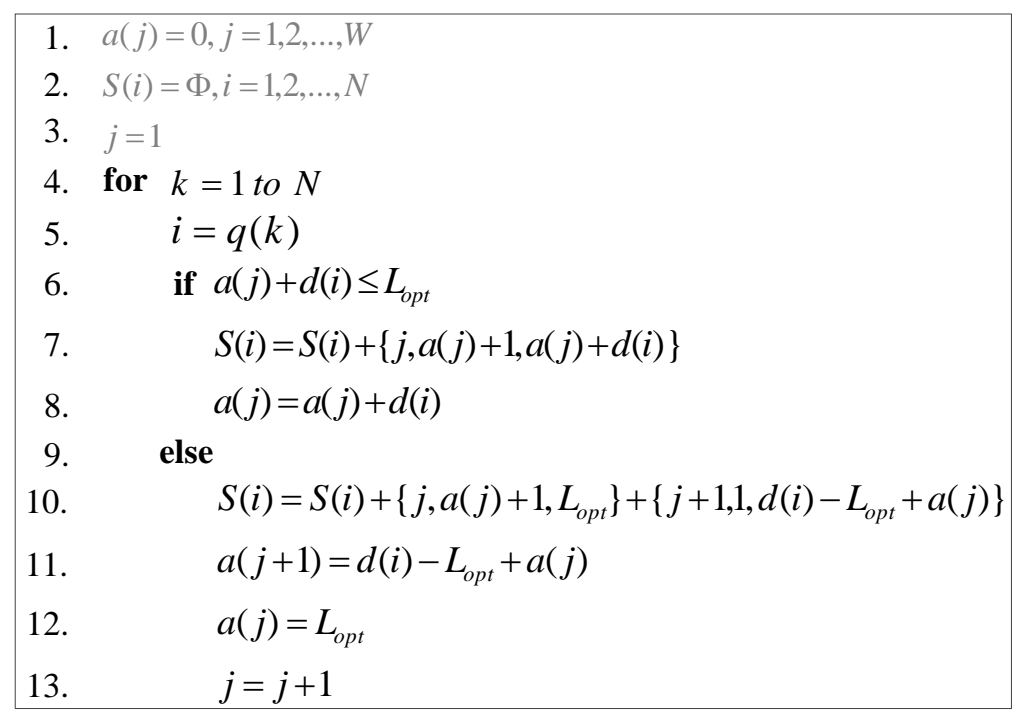

Fig. 5 The pseudo-code of the step 3) in scheduling WDM/TDM subsystems

As mentioned above, the subsystem's demand needs to be sorted with descending order firstly. The reason is that it can avoid the wavelength-switched influence on the proposed algorithm. Here, an example is used to illustrate the 
situation. We suppose that there are two wavelengths ( $w 1$ and $w 2$ ) and three subsystems ( $s 1, s 2$ and $s 3$ ). Their demands are two, five and three time slots respectively. Different scheduling could lead to different results. If the subsystems are scheduled sequentially, the wavelength-switched influence will appear. The sequential scheduling result is shown in Fig. 6(a). As described in Fig. 6(a), s2 is instantaneously changed from $w 2$ to $w 1$, which is impossible for tunable laser. Thus, in this scheduling scheme, $s 2$ will have to use the scheduled time slot to perform the wavelength switching and this in turn will degrade the performance. However, if we use the proposed scheduling algorithm, i.e., sorting the $d(i)$ with the descending order, the result will be different. As shown in Fig. 6(b), s2 occupies the whole wavelength, which means the wavelength switching can be avoided.

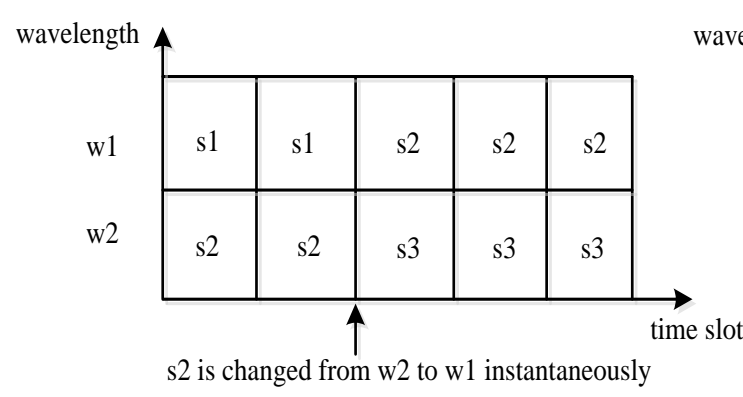

(a)

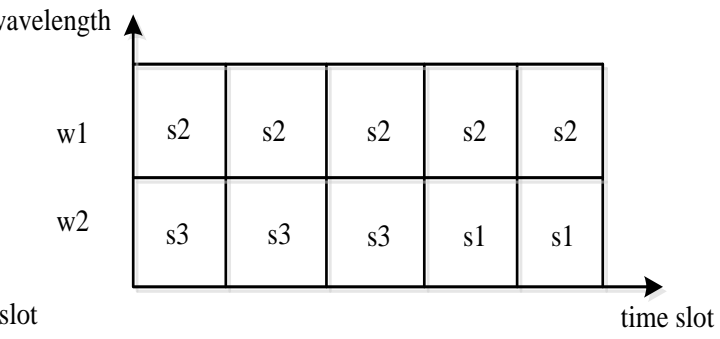

(b)

Fig. 6 An example of scheduling WDM/TDM subsystems, including (a) sequential scheduling and (b) the proposed multi-channel scheduling

\section{B. WDM/OFDM subsystems}

To the WDM/OFDM subsystems, we assume the transceiver in each FCN independently uses a wavelength to transmit upstream traffic to the $\mathrm{CN}$ because of the optical beat noise [13]. Therefore, the multi-channel scheduling is aimed at subcarrier scheduling here. Since each subsystem can use multiple subcarriers at the same time, the "non-overlapping constraint" will not exist in the WDM/OFDM subsystems. Therefore, the optimal scheduling length can directly be defined as follows:

$$
L_{o p t}=\left\lceil\frac{\sum_{i=1}^{N} d(i)}{W}\right\rceil
$$

The multi-channel scheduling algorithm works as follows: 1) calculate the optimal scheduling length $L_{\text {opt }}$. 2) assign each subsystem to channels. The pseudo-code of the step 2) is shown in Fig. 7. It is easy to see that Fig. 7 is similar to Fig. 5 except that the line 5 in Fig. 5 doesn't exist in Fig. 7. This is because the 
subsystems are scheduled sequentially here instead of according to the descending order of their demand. Therefore, the mapping from $k$ to $i$ is omitted.

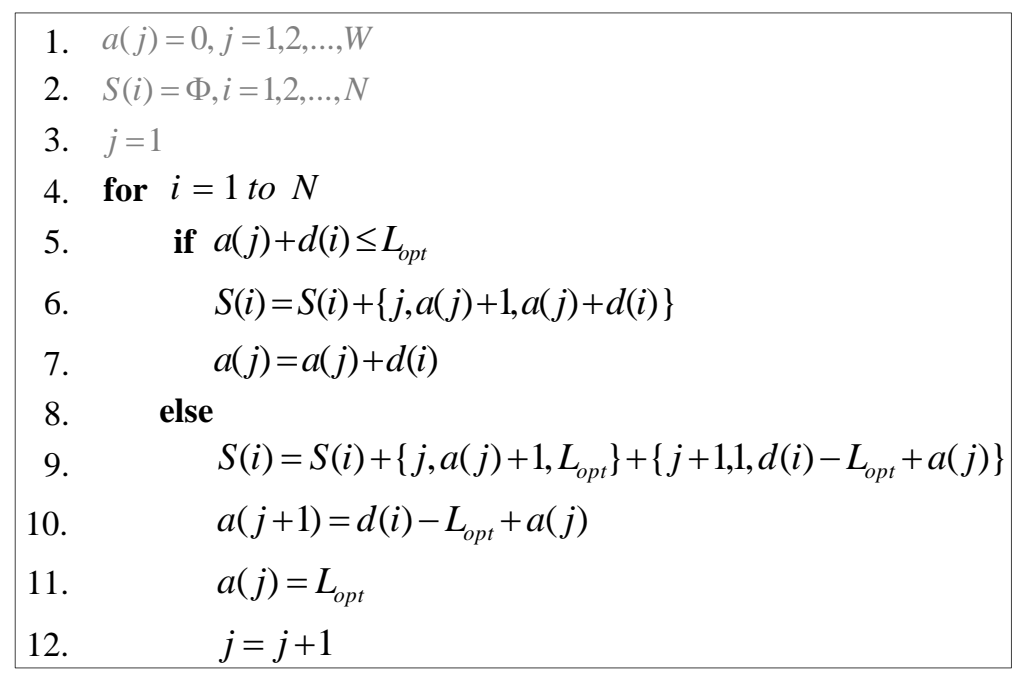

Fig. 7 The pseudo-code of the step 2) in scheduling WDM/OFDM subsystems

To distinguish the scheduling of WDM/TDM subsystems and the scheduling of WDM/OFDM subsystems, we could denote the former as WDM/TDM Subsystems (WTS) and the latter as WDM/OFDM Subsystems (WOS). It is easy to observe that the optimal scheduling length of WOS is not bigger than that of WTS. Therefore, WOS always has same or higher channel-utilized ratio than WTS. The channel-utilized ratio of WOS is calculated as follows:

$$
U=\frac{\sum_{i=1}^{N} d(i)}{\left\lceil\frac{\sum_{i=1}^{N} d(i)}{W}\right\rceil \cdot W}
$$

Compared with WTS, WOS doesn't need to sort $d(i)$ with the descending order. The reason is that each subsystem is allocated with an independent wavelength. In other words, the wavelength-switched influence in the WDM/TDM subsystems doesn't exist in the WDM/OFDM subsystems. If we assume that there are two subcarriers ( $c 1$ and $c 2)$ and three subsystems ( $s 1, s 2$ and $s 3)$. Their demands are two, five and three time slots respectively. The scheduling result is shown in Fig. 8. Compared with the result in Fig. 6(b), the channel-utilized ratio of WOS is the same as that of WTS because the $\left\lceil\sum_{i=1}^{N} d(i) / W\right\rceil$ is equal to $\max (d(i))$ at this time. 


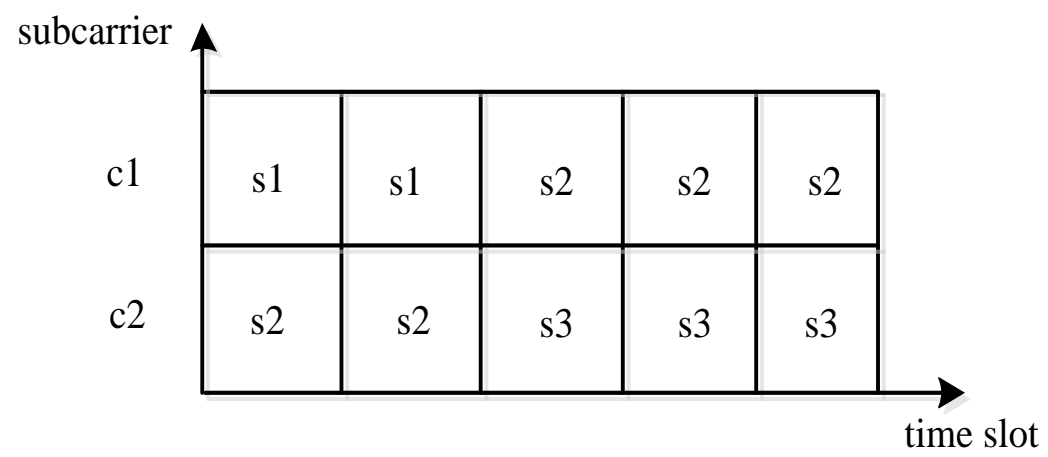

Fig. 8 An example of scheduling WDM/OFDM subsystems with the proposed algorithm

\section{Performance evaluation}

The performance of the proposed algorithm is evaluated through MATLAB. In order to show how the proposed algorithm achieves the optimal scheduling, we compare the proposed algorithm with the sequential scheduling algorithm (SSA) that sequentially assigns each subsystem onto a channel one-by-one. In simulations, the demand of each subsystem $(d(i))$ is uniformly distributed in the range of $\left[d_{\min }, d_{\max }\right]$. In addition, to facilitate illustration, the number of available channels $(W)$ is set to 10 in all simulations as a representative example. Two cases are investigated. In case 1, the number of subsystems $(N)$ is fixed at 10 and 15 . The minimum demand $\left(d_{\min }\right)$ is fixed at 3 , but the maximum demand ( $\left.d_{\max }\right)$ varies from 10 to 30 with an increment of

2. The larger $d_{\max }$, the larger the discrepancy among the subsystems' demand. Therefore, the purpose of this case is to study the impact of the demand discrepancy on the channel-utilized ratio. In other words, case 1 can be applied to the circumstance of unbalanced load among various subsystems. In case $2, d_{\min }$ is fixed at $3, d_{\max }$ is fixed at 10 and 30. However, the number of subsystems changes from 10 to 100 with an increment of 10. The purpose of this case is to study the impact of the number of subsystems on the channel-utilized ratio. Namely, case 2 can be applied to the situation of increasing subsystems (i.e., network expansion). 


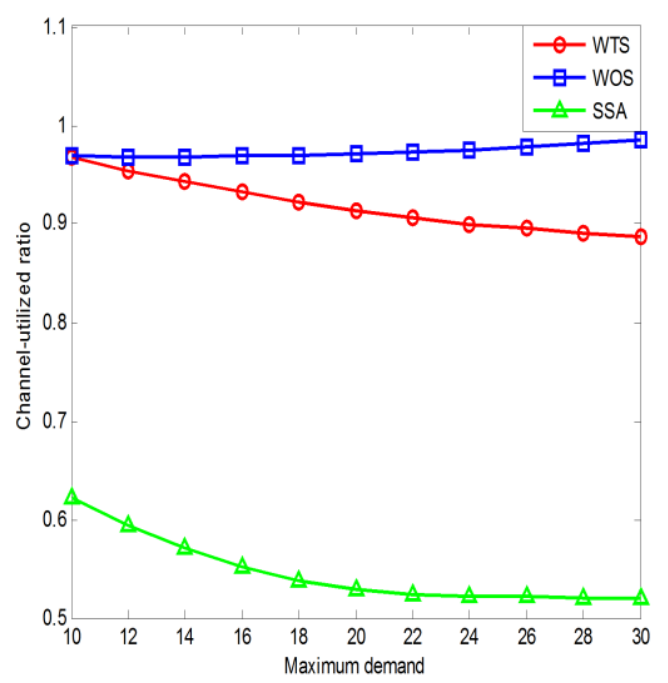

(a) 10 subsystems

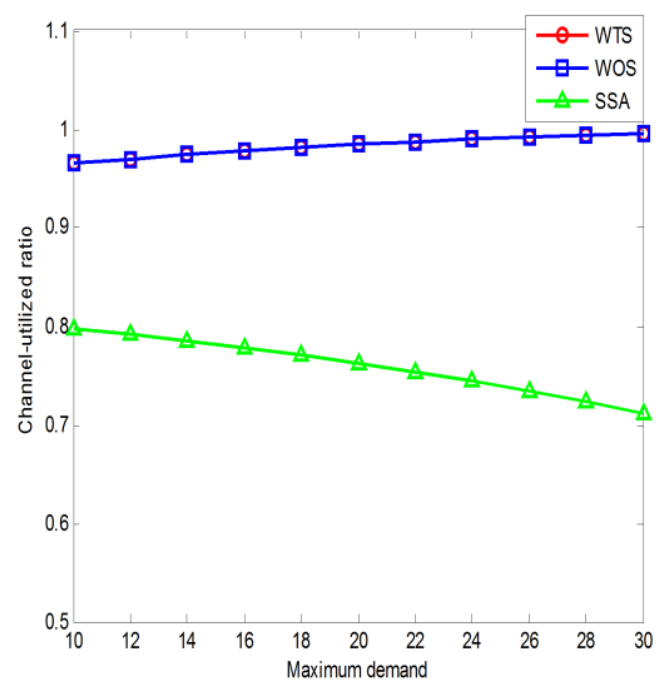

(b) 15 subsystems

Fig. 9 Channel-utilized ratio versus maximum demand with (a) 10 subsystems (b)

$$
15 \text { subsystems }
$$

Fig. 9 shows the channel-utilized ratio of the first case. From the Fig. 9, we can see that the more subsystems to complete the channel, the higher channel-utilized ratio will be achieved. The reason is that the more subsystems lead to lower $d(i)$ discrepancy among them and accordingly higher utilization. In addition, with the increase of $d_{\max }$, the channel-utilized ratio of SSA decreases because the higher $d_{\max }$ implies the larger discrepancy among $d(i)$ and accordingly more time slots will be wasted under SSA. Finally, SSA has the lowest channel-utilized ratio. WTS and WOS achieve much higher utilization than SSA. WOS has the highest utilization since it allows subsystems to occupy more than one channel simultaneously. When there are more subsystems (i.e., Fig. 9(b)), the channel-utilized ratio of WTS is the same as that of WOS. The reason is that the more subsystems, the less deviation among $d(i)$ and therefore $\max (d(i))$ will be equal to $\left\lceil\sum_{i=1}^{N} d(i) / W\right\rceil$ at this time. 


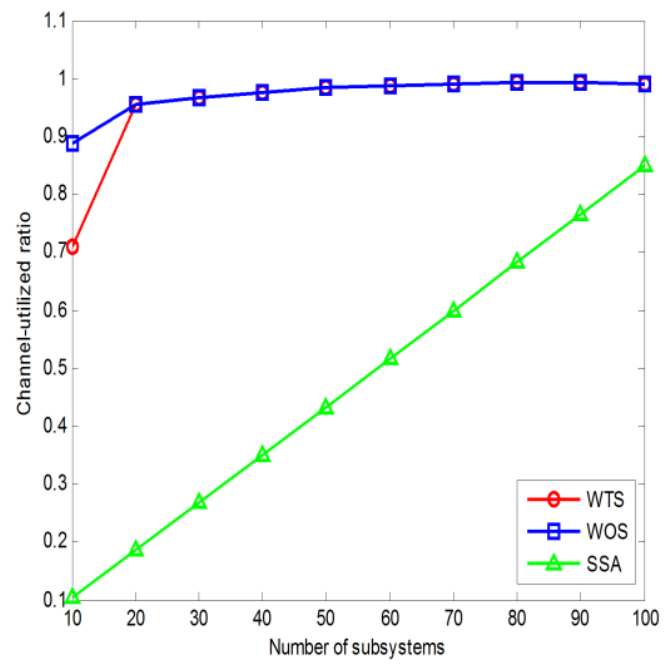

(a) Maximum demand $=10$

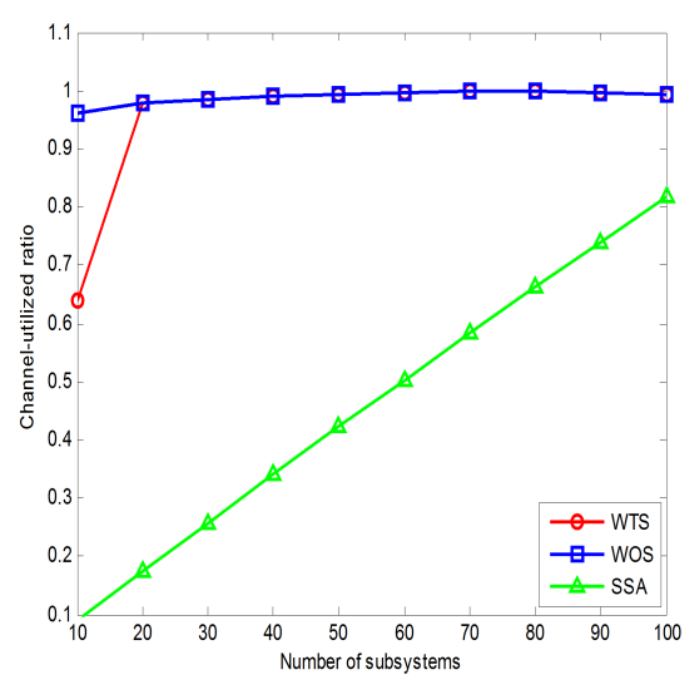

(b) Maximum demand $=30$

Fig. 10 Channel-utilized ratio versus number of subsystems with (a) Maximum demand=10 (b) Maximum demand=30

Fig. 10 depicts the channel-utilized ratio of the second case. It can be seen that each algorithm obtains increasing utilization when the number of subsystems increases. The reason is that the demand discrepancy among all subsystems will decrease with the increased number of subsystems. In turn, it is more likely to make each channel have similar length and improve the channel-utilized ratio accordingly. In addition, WTS and WOS always achieve much higher channel-utilized ratio than SSA. Moreover, when there are certain enough subsystems, the channel-utilized ratio of WTS is identical to that of WOS because the scheduling length of WTS is equal to that of WOS at this time. Finally, we can find that the channel-utilized ratio of WTS increases obviously when the subsystems increase from 10 to 20 . The reason can be stated as follows: as mentioned above, the number of channels is set to 10 in all simulations. When the number of subsystems is 10 , the number of subsystems is equal to the number of channels. In other words, each channel will be occupied by one subsystem in average. However, when the number of subsystems is 20 , the number of subsystems is twice as many as the number of channels. In other words, each channel will be occupied by two subsystems in average. Therefore, when the demand distribution of each subsystem is the same in these two situations, the channel-utilized ratio of the latter will be obviously higher than that of the former. Namely, there will be an obvious increase in channel-utilized ratio when subsystems increase from 10 to 20 .

Compared with WOS and SSA, WTS has the probability that some channels may be unoccupied after scheduling. These channels are referred to as idle channels. Therefore, FCNs can switch off transmitters corresponding to these idle channels to reduce energy consumption. To present the number of channels actually used in WTS, 
$W_{\text {actual }}$ is defined as follows:

$$
W_{\text {actual }}=\left\lceil\frac{\sum_{i=1}^{N} d(i)}{L_{\text {opt }}}\right\rceil
$$

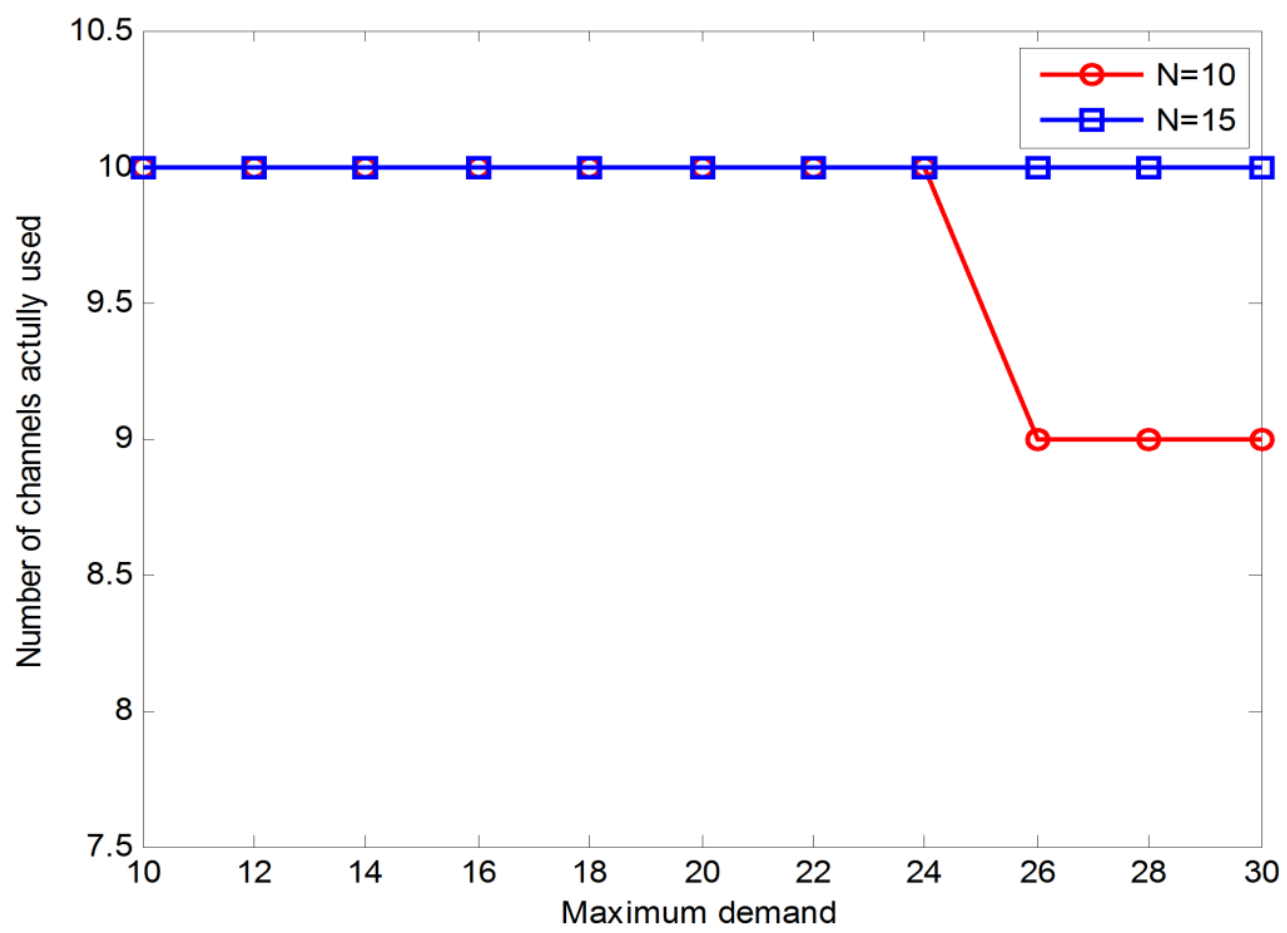

Fig. 11 Number of channels actually used versus maximum demand

Fig. 11 shows the number of channels actually used in the first case. From the Fig. 11 , we can see that when $N$ is $10, W_{\text {actual }}$ decreases with the increase of $d_{\max }$. The reason is that the higher $d_{\max }$, the larger discrepancy among $d(i)$. According to Eq. (3), this will lead to the increase of optimal scheduling length. Thus, $W_{\text {actual }}$ will decrease according to Eq. (7). When $N$ is $15, W_{\text {actual }}$ is always equal to 10 . At this time, since the number of subsystems is bigger than the number of channels, all the channels will be occupied by these subsystems. 


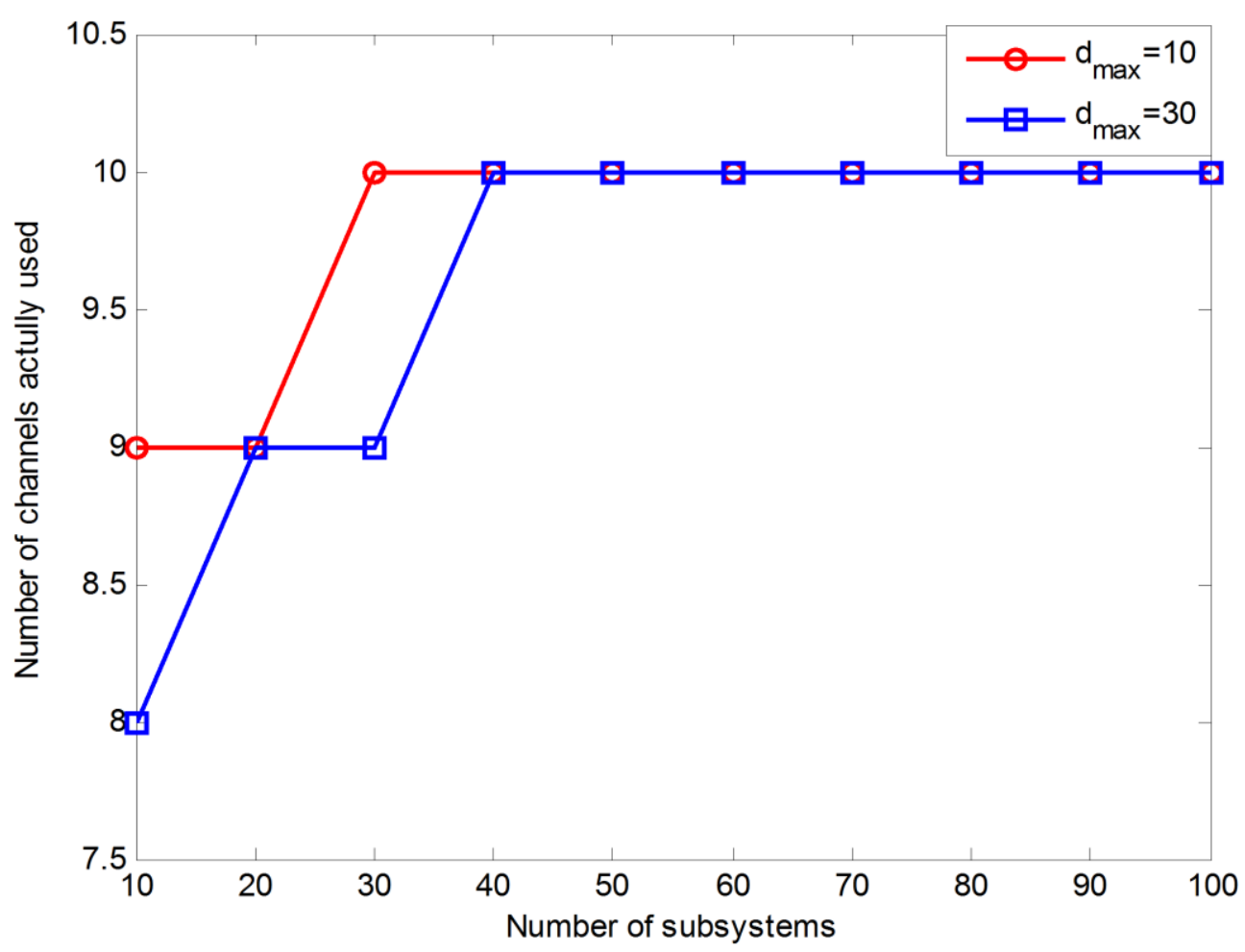

Fig. 12 Number of channels actually used versus number of subsystems

Fig. 12 describes the number of channels actually used in the second case. It is obvious that more channels will be occupied with the increase of subsystems. When $d_{\max }$ is 10 , the optimal scheduling length is smaller than the situation when $d_{\max }$ is

30. This is because smaller $d_{\max }$ leads to smaller discrepancy and then results in smaller scheduling length. Therefore, according to Eq. (7), it is clear that $W_{\text {actual }}$ will firstly tend to 10 when $d_{\max }$ is equal to 10 .

\section{Conclusion}

Multi-channel scheduling algorithm for MS-VPON in MAON is proposed in this paper. To the MS-VPON scenario, the adaptive transceiver is designed to support multi-channel scheduling in the metro segment with the reuse of original deployment in the access segment. With the adaptive transceiver, the multi-channel scheduling algorithm is applied to schedule wavelength and subcarrier resource in the WDM/TDM subsystems and WDM/OFDM subsystems respectively. According to the algorithm, the centralized management is used. The channel-utilized ratio is maximized. The simulation results show a good performance of the proposed algorithm in the channel-utilized ratio compared with sequential scheduling algorithm. 


\section{Acknowledgment}

This work is supported by Programs of National Science Foundation of China (No. 61132004, 61275073 and 61420106011), Shanghai Science and Technology Development Funds (No. 13JC1402600, 14511100100, 15511105400 and 15530500600) and Shanghai Leading Academic Discipline Project (No. S30108).

\section{Reference}

[1] A. Tzanakaki, M. P. Anastasopoulos, G. S. Zervas, et al. Virtualization of Heterogeneous Wireless-Optical Network and IT Infrastructures in Support of Cloud and Mobile Cloud Services. IEEE Communications Magazine, 2013, 51(8): 155-161.

[2] T. Kuri, H. Harai, N. Wada, et al. Adaptable Access System: Pursuit of Ideal Future Access System Architecture. IEEE Network, 2012, 26(2): 42-48.

[3] A. Khan, A. Zugenmaier, D. Jurca, et al. Network Virtualization: A Hypervisor for the Internet? IEEE Communications Magazine, 2012, 50(1): 136-143.

[4] C. Fei, W. Kehe, C. Wei, et al. The research and implementation of the VPN gateway based on SSL. International Conference on Computational and Information Sciences (ICCIS), 2013: 1376-1379.

[5] R. Yadav. Passive-Optical-Network- (PON-) Based Converged Access Network. Journal of Optical Communications and Networking, 2012, 4(11): B124-B130.

[6] T. Su, Y. Tian, E. Wong, et al. All-optical virtual private network in passive optical networks. Laser \& Photonics Reviews, 2008, 2(6): 460-479.

[7] W. Wei, T. Wang, C. Qiao. Resource Provisioning for Orthogonal Frequency Division Multiple Access (OFDMA)-based Virtual Passive Optical Networks (VPON). Optical Fiber Communication/National Fiber Optic Engineers Conference (OFC/NFOEC), 2008: 1-3.

[8] L. Zhou, H. Lin, G. Peng, et al. A Novel Optical Access Network: Software-Defined FlexPON. OptoElectronics and Communication Conference 
and Australian Conference on Optical Fiber Technology (OECC/ACOFT), 2014: 688-690.

[9] L. Zhou, H. Lin, G. Peng, et al. Demonstration of Software-Defined FlexPON. International Conference on Optical Network Design and Modeling (ONDM), 2014: 37-42.

[10]H. Lin, G. Peng, L Zhou, et al. Software-Defined FlexPON. World Telecommunications Congress (WTC), 2014: 1-3.

[11]X. Cheng, Z. Xu, Y. Yeo et al. Wavelength-agile Hybrid Passive Optical Networks with Dynamic ONU Re-Grouping Functionality. International Conference on Information, Communications and Signal Processing (ICICS), 2011: 1-4.

[12]X. Cheng, Y. Yeo, Z. Xu et al. Hybrid WDM/TDM Passive Optical Network with Dynamic Virtual PON (VPON) Capability. European Conference and Exhibition on Optical Communication (ECOC), 2010: 1-3.

[13]W. Wei, J. Hu, D. Qian et al. PONIARD: A Programmable Optical Networking Infrastructure for Advanced Research and Development of Future Internet. Journal of Lightwave Technology, 2009, 27(3): 233-242.

[14]K. Kanonakis, N. Cvijectic, I. Tomkos et al. Dynamic Software-Defined Resource Optimization in Next-Generation Optical Access Enabled by OFDMA-Based Meta-MAC Provisioning. Journal of Lightwave Technology, 2013, 31(14): 2296-2306.

[15]N. Minato, S. Kobayashi, K. Sasaki, et al. Design of Hybrid WDM/OCDM Add/Drop Filters and Its Experimental Demonstration for Passive Routing in Metropolitan and Access Integrated Network. Journal of Lightwave Technology, 2014, 32 (6): 1120-1131.

[16]B. Schrenk, A. Poppe, M. Stierle, et al. Fully-Passive Optical Switch Introducing Dynamicity and Flexibility to Metro-Access. IEEE Photonics Technology Letters, 2015, 27(5): 486-489.

[17]A. Ahmed and A. Shami. RPR-EPON-WiMAX Hybrid Network: A Solution for Access and Metro Networks. Journal of Optical Communications and Networking, 2012, 4(3): 173-188. 
[18]F. Aurzada, M. Scheutzow, M. Reisslein, et al. Capacity and Delay Analysis of Next-Generation Passive Optical Networks (NG-PONs), IEEE Transaction on Communications, 2011, 59(5): 1378-1388.

[19]W. Jin, C. Zhang, C. Chen, et al. Scalable and Reconfigurable All-Optical VPN for OFDM-Based Metro-Access Integrated Network. Journal of Lightwave Technology, 2014, 32 (2): 318-325.

[20]X. Hu, X. Chen, Z. Zhang, et al. Dynamic Wavelength and Bandwidth Allocation in Flexible TWDM Optical Access Network. IEEE Communications Letters, 2014, 18 (12): 2113-2116. 